\title{
Objectively measured physical activity and all cause mortality: a systematic review and meta-analysis
}

Rema Ramakrishnan, ${ }^{1,2}$ Jian-Rong He, ${ }^{1,3}$ Anne-Louise Ponsonby, ${ }^{4,5,6}$ Mark Woodward, ${ }^{1,7,8}$ Kazem Rahimi, ${ }^{1,9,10,11}$ Steven N. Blair, ${ }^{12,13}$ Terence Dwyer ${ }^{14,15}$

${ }^{1}$ The George Institute for Global Health, Nuffield Department of Women's and Reproductive Health, University of Oxford, Oxford, UK

${ }^{2}$ University of New South Wales, Faculty of Medicine, Sydney, Australia

${ }^{3}$ Division of Birth Cohort Study, Guangzhou Women and Children's Medical Center, Guangzhou Medical University, Guangzhou, China.

${ }^{4}$ The Florey Institute for Neuroscience and Mental Health, Melbourne, Australia

${ }^{5}$ Department of Environmental and Genetic Epidemiology, Murdoch Children's Research Institute, Royal Children's Hospital, University of Melbourne, Melbourne, Australia ${ }^{6}$ National Centre for Epidemiology and Population Health, Australian National University ${ }^{7}$ The George Institute for Global Health, University of New South Wales, Sydney, Australia ${ }^{8}$ Department of Epidemiology, Johns Hopkins University, Baltimore, USA

${ }^{9}$ National Institute for Health Research Oxford Biomedical Research Centre, University of Oxford, Oxford, United Kingdom

${ }^{10}$ Deep Medicine, Oxford Martin School, University of Oxford, United Kingdom

${ }^{11}$ Oxford University Hospitals NHS Foundation Trust, Oxford, United Kingdom

${ }^{12}$ Department of Exercise Science, Arnold School of Public Health, University of South Carolina, Columbia, South Carolina, USA

${ }^{13}$ Department of Epidemiology and Biostatistics, Arnold School of Public Health, University of South Carolina, Columbia, South Carolina, USA

${ }^{14}$ Nuffield Department of Women's \& Reproductive Health, University of Oxford, Oxford, $\mathrm{UK}$

${ }^{15}$ Murdoch Children's Research Institute, Melbourne, Australia

Corresponding Author:

Name: $\quad$ Terence Dwyer

Address: $\quad$ Nuffield Department of Women's \& Reproductive Health, University of Oxford Level 3, Women's Centre

John Radcliffe Hospital 
Oxford OX3 9DU

Affiliations: i) Nuffield Department of Women's \& Reproductive Health, University of Oxford, Oxford, UK;

ii) Murdoch Children's Research Institute, Melbourne, Australia

Email: $\quad$ terence.dwyer@wrh.ox.ac.uk

Keywords: mortality, physical activity, objectively measured

Abstract word count: 250

Manuscript word count: 3,810 


\begin{abstract}
Current physical activity recommendations have been based on evidence from systematic reviews of questionnaire-based data. Questionnaire-based physical activity data are subject to both random and non-random error. If the estimated association between physical activity and health outcomes was different when a more accurate, objective measure was used, this would have important health policy implications for physical activity. We conducted a systematic review and meta-analysis of published cohort studies that investigated the association between an objective measure of physical activity and all cause mortality. We searched PubMed, Scopus, Embase, Cochrane library, and SPORTDiscus for prospective cohort studies that examined the association between objectively measured (accelerometer, pedometer, or doubly labeled water method) physical activity and mortality in adults aged $\geq 18$ years, of either sex. Summary hazard ratios and $95 \%$ confidence interval $[\mathrm{CI}] \mathrm{s}$ were computed using random-effects models. Thirty-three articles from 15 cohort studies were identified that together ascertained 3,903 deaths. The mean years of follow-up ranged from 2.3-14.2 years. Individuals in the highest category of light, moderate-to-vigorous, and total physical activity had $40 \%$ (95\%CI $20 \%$ to $55 \%$ ), $56 \%$ (95\%CI $41 \%$ to $67 \%$ ), and $67 \%$ (95\% CI $57 \%$ to $75 \%$ ), respectively, lower risk for mortality compared to individuals in the lowest category of light, moderate-to-vigorous, and total physical activity. The summary hazard ratio for objectively measured physical activity and all cause mortality is lower than previously estimated from questionnaire based studies. Current recommendations for physical activity that are based on subjective measurement may underestimate the true reduction in mortality risk associated with physical activity.
\end{abstract}

Keywords: accelerometry; mortality; exercise; objectively measured; meta-analysis; systematic review 


\section{INTRODUCTION}

Leisure time physical activity (PA) is inversely associated with mortality, and with incidence of non-communicable diseases, but with most of the potential benefit gained from relatively modest amounts of activity. In a meta-analysis, Woodcock et $\mathrm{al}^{1}$ estimated that if a sedentary individual increased light to moderate activity in leisure time from none to only 11 metabolic equivalent (MET) hours/ week, or 2.5 hours/week of activity with an intensity equivalent to brisk walking, they would reduce their mortality risk by $19 \%$. A further increase to $31 \mathrm{MET}$ hours/week, or 7 hours per week would only reduce risk of death by a further five percentage points. ${ }^{1}$ Samitz et $\mathrm{al}^{2}$ similarly found hazard ratio (HR)s of 0.86 and 0.74 , respectively, for $2 \frac{1}{2}$ hours and five hours of moderate-to-vigorous PA (MVPA) per week compared to the lowest level of activity (median $11 \mathrm{~min} /$ week).

The results from these systematic reviews underpin the population PA targets recommended by the U.S. Department of Health and Human Services and the Chief Medical Officer, UK- that is, at least, 21/2 hours of moderate PA such as brisk walking per week. ${ }^{3,4}$ However, the validity of these recommendations depends on the accuracy of the measurement used to capture PA in the studies included in the systematic reviews - in each case, questionnaires. While questionnaires capture this exposure to some extent, they have been shown to be affected by a material level of both random and non-random error ${ }^{5,6}$ which can be minimised using an objective measure of PA such as doubly labeled water, accelerometer, or pedometer.

A meta-analysis of 12 articles from 11 cohort studies among adults aged $\geq 60$ years of age found device-measured light PA, MVPA, and total PA to be associated with 1.93, 2.66, and 3.43 higher risk for all cause mortality among participants who were least active compared with the most active group. ${ }^{7}$ A systematic review of five studies found $6 \%-36 \%$ reduction in risk for mortality for each 1,000 increase in daily step count measured by pedometer or accelerometer. ${ }^{8}$ An individual participant data (IPD) meta-analysis that examined accelerometer measured PA and all cause mortality using data from eight studies (six published and two unpublished) found 73\%, 62\%, and $48 \%$ lower risk when comparing the highest to lowest category of total, light, and MVPA, respectively, ${ }^{9}$ which is much lower than what has been found for questionnaire-based studies. To add to this evidence, we conducted a systematic review and meta-analysis of published cohort studies that reported the association between an objective measure of PA and all cause mortality among adults $\geq 18$ years of age. This study that included the largest number of published studies on this topic till 
date had no restrictions to type of device used to measure PA, unit of measurement, or age group among adults.

\section{METHODS}

In this systematic review and meta-analysis, we followed the guidelines in the Preferred Reporting Items for Systematic Reviews and Meta-Analyses (PRISMA) statement. Five electronic databases, Medline via PubMed, Scopus, Embase via Ovid, Cochrane library, and SPORTDiscus, were searched up to and including August 31, 2020 with no language restrictions. We searched the bibliographies of included records to identify additional studies. We did not contact the corresponding authors of papers because we did not require any additional information/clarification. The search terms for all the databases included, accelerometry, accelero*, actigraphy, actigra*, objectively assessed physical activity, objectively measured physical activity, pedometer, doubly labelled water, doubly labeled water, and mortality. Details of the search strategy are given in Supplementary Table 1.

Studies were considered eligible for inclusion if i) they were prospective cohort studies that measured PA objectively by accelerometer, pedometer, or the doubly labeled water method, ii) they investigated the association between objectively assessed light, moderate, vigorous, or moderate-to-vigorous, or as steps, and mortality from any cause, and iii) the participants were adults aged 18 years and older. We excluded studies that examined the association in specific disease populations (for example, patients with cancer, type 2 diabetes, or chronic heart failure). Only original research articles were included; consequently, editorials, commentaries, reviews, conference abstracts, letters, or research briefs were excluded.

We used a data extraction form in Microsoft Excel that had been developed a priori by RR. The information extracted were: study name, study design and population, country, sample size, mean age, percentage of male participants, length and person-years of follow-up, mortality rate, number of deaths, PA measurement instrument, unit of measurement, PA definition, mean PA at baseline, PA categories, early deaths included (yes/no), adjustment factors, subgroup analysis (yes/no), the grouping variable for subgroup analysis, and the point estimate (hazard ratio[HR]/odds ratio/relative risk) with its lower and upper 95\% confidence limits. Most of the studies reported the estimates in quantiles (tertiles, quartiles, and quintiles), few treated PA as a continuous measure. If a study reported relative risk $^{10}$ we treated it as HR. The only study that reported log odds ratio ${ }^{11}$ did not have data that could be harmonised. If, instead of HRs, the observed and logrank expected events were reported in 
each exposure category, ${ }^{12}$ then the method/s reported by Tierney et al ${ }^{13}$ was used to compute the HRs. For studies that reported only p-values for statistical significance, ${ }^{11,14}$ the confidence intervals were computed using the Altman and Bland method. ${ }^{15}$ For each PA category (light, moderate-to-vigorous, and total), if there were multiple publications from the same cohort, we selected the one with the longest follow-up; if two studies had the same length of followup then the one with the largest sample size or had data that could be harmonized was included in the meta-analysis. If a study reported separate estimates for low and high light PA then the latter was chosen for the analyses. If there were discrepancies in the estimates in the text and tables, the numbers in tables were included in the review and analysis. One reviewer (RR) selected and extracted the datadata, and a second reviewer (JH) checked the data for accuracy; inconsistencies were resolved through discussions.

Two reviewers (RR and $\mathrm{JH})$ independently evaluated the quality of included studies using the Newcastle Ottawa Scale. For cohort studies, this scale has a high inter-rater reliability, $0.94 .{ }^{16}$ In this scale, the quality of a study is assessed using three domains selection of exposed and non-exposed cohort, comparability of cohorts based on design/analysis, and outcome assessment. A star is awarded for each item within the selection and outcome domains and a maximum of two stars are awarded for comparability. ${ }^{17}$ For the comparability domain, we considered comorbidities/self-rated health status to be the most important confounder due to its association with both mortality and PA. The maximum number of stars that a single study could accomplish was nine. We rated the quality of studies as high, medium, or low based on $8-9,5-7$, and $<5$ stars, respectively. Any discrepancy in rating was resolved through discussions between the reviewers (RR and JH). Details of evaluation of measures of quality are given in the Supplementary text.

Light, moderate-to-vigorous, and total PA were defined as reported in the studies. If the study reported steps/day, or total accelerometer counts, then these totals were used to define total PA. For each category of PA (light, moderate-to-vigorous, and total), we compared HRs for the highest to that of the lowest category (Supplementary Table 2). For studies that compared the lowest to the highest category, we took the reciprocal of the point estimate and used that for the meta-analysis.

We used the DerSimonian and Laird random-effects model ${ }^{18}$ to pool estimates for the association between light, moderate-to-vigorous, and total PA and mortality and for doseresponse, subgroup, and sensitivity analyses. We conducted separate analysis for five studies that measured total PA in steps/day as a continuous measure. ${ }^{12,19-22}$ We examined the doseresponse association between MVPA and all cause mortality by restricted cubic spline 
analysis wherein three knots were specified at 0.2, 0.4, and 1.0 hour/day of MVPA. The Wald test was used to assess for non-linearity by testing the point estimate of the second spline was equal to zero. ${ }^{23} \mathrm{We}$ assessed statistical heterogeneity among studies included in the analysis using the $\mathrm{I}^{2}$ statistic. For the calculation of the $\mathrm{I}^{2}$ statistic, we used the natural $\log$ of $\mathrm{HR}$ and 95\% CI in a random-effects model. The degree of heterogeneity was categorised as low, moderate, and high based on $\mathrm{I}^{2}$ values of $25 \%, 50 \%$, and $75 \%$, respectively. ${ }^{24}$ Publication bias and small study effects were assessed through funnel plot and Egger's test for funnel plot asymmetry for exposure category with at least ten studies.

We conducted sensitivity analysis to assess the impact of outliers (extreme values for the reference category) on the estimates and studies with different definitions of exposure categories: for example, to examine the association between total PA and mortality, we excluded studies that defined the exposure as steps/day and only studies that measured minutes/day were included. We also assessed sensitivity of the estimates to exclusion of early deaths through analysis of studies that reported hazard ratios/relative risks after excluding these participants. Finally, we investigated if the estimates varied by sex, age ( $>60$ years vs $\leq 60$ years), study quality, and years of follow-up ( $<5$ years vs $\geq 5$ years).

All analyses were conducted using STATA version 14.0 (StataCorp, College Station, TX, USA).

\section{RESULTS}

We retrieved 1,129 records from a search of studies published up until June 17, 2018 from PubMed, Scopus, Embase through Ovid, the Cochrane library, and SPORTSdiscus. An updated search up until August 31, 2020 unearthed additional 367 records appending the total to 1,496. Exclusion of duplicate records resulted in 1,256 records for further assessment. Screening of the title and abstract resulted in 51 articles. Eighteen articles were excluded after full texts of the studies were examined resulting in 33 articles ${ }^{10-12,14,19-22,25-49}$ from 15 cohort studies for the systematic review and meta-analyses (Supplementary Figure 1). Out of 33 articles, 18 were from the National Health and Nutrition Examination Survey (NHANES)

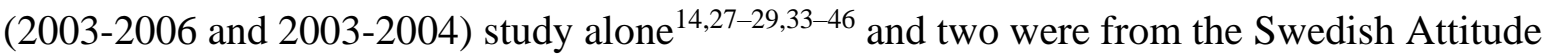
Behaviour and Change study ${ }^{31,47}$ Though we included these studies for the systematic review, for the meta-analysis we included only two NHANES studies ${ }^{27,28}$ - one for light PA and MVPA and another for total PA- and one paper from the Swedish Attitude Behaviour and Change study ${ }^{31,47}$. We included only these studies because they had exposures that could be 
categorized into highest versus lowest category and had longer follow-up periods followed by larger sample size.

The descriptive information for 33 articles from 15 cohort studies is presented in Table 1. The 15 studies included 141,582 participants with mean age at baseline ranging from $48.4^{27}$ years to 85 years. ${ }^{11}$ Two studies were each conducted exclusively among men ${ }^{20,30}$ and women ${ }^{10,22}$ whereas in the remaining studies there was a preponderance of female participants. Out of the 15 studies, six were conducted in the US, ${ }^{10,14,22,25,27-30,33-46,48}$ three in the UK, ${ }^{19,20,32}$ and one each in Australia, ${ }^{12}$ Brazil,${ }^{49}$ Canada,${ }^{11}$ Japan,${ }^{21}$ Norway, ${ }^{26}$ and Sweden. ${ }^{31,47}$ The mean/median follow-up fell within the range 2.3 years-14.2 years, whereas the mortality rate ranged from 5 per 10,000 person-years $^{32}$ to 850 per 10,000 person-years. $^{12}$ 
Table 1. Characteristics of the cohort studies included in the systematic review

\begin{tabular}{|c|c|c|c|c|c|c|c|c|c|c|}
\hline Study & Author & Country & $\begin{array}{l}\text { Sample } \\
\text { size }\end{array}$ & $\begin{array}{l}\text { Age } \\
\text { (years) }\end{array}$ & Male\% & $\begin{array}{l}\text { Mean: } \\
\text { Follow- } \\
\text { up } \\
\text { length } \\
\text { (years) }\end{array}$ & $\begin{array}{l}\text { Mortality } \\
\text { rate per } \\
10,000 \\
\text { PY }\end{array}$ & $\begin{array}{l}\text { Physical } \\
\text { Activity } \\
\text { Measurement } \\
\text { Instrument }\end{array}$ & $\begin{array}{l}\text { Mean PA } \\
\text { (baseline) }\end{array}$ & Covariates \\
\hline $\begin{array}{l}70 \text {-years olds in } \\
\text { Niigata city, Japan in } \\
1998\end{array}$ & $\begin{array}{l}\text { Yamamoto, } \\
2018^{21}\end{array}$ & Japan & 419 & 71 & 54.4 & 9.8 & 185 & Pedometer & $\begin{array}{l}6,470 \\
\text { steps/day }\end{array}$ & $\begin{array}{l}\text { Sex, body mass index, } \\
\text { cigarette smoking, } \\
\text { alcohol intake, and } \\
\text { medication use }\end{array}$ \\
\hline $\begin{array}{l}\text { Baltimore } \\
\text { Longitudinal Study } \\
\text { of Aging }\end{array}$ & $\begin{array}{l}\text { Wanigatunga, } \\
2019^{48}\end{array}$ & USA & 548 & 75.8 & 52.2 & 4.4 & 253 & Accelerometer & $\begin{array}{l}\text { Total PA: } \\
\text { 5.6 } \\
\text { hours/day }\end{array}$ & $\begin{array}{l}\text { Age, sex, race/ethnicity, } \\
\text { body mass index, } \\
\text { smoking history, } \\
\text { currently working for } \\
\text { pay, self-reported health, } \\
\text { grip strength, usual gait } \\
\text { speed, comorbidities, } \\
\text { and activity monitor } \\
\text { wear days. }\end{array}$ \\
\hline Como Vai study & $\begin{array}{l}\text { Bielemann, } \\
2020^{49}\end{array}$ & Brazil & 971 & $\begin{array}{l}60-69 \\
\text { years: } \\
51.1 \%, \\
70-79 \\
\text { years: } \\
34.7 \%, \\
\geq 80 \\
\text { years } \\
14.2 \%\end{array}$ & 37.8 & 2.6 & 300 & Accelerometer & $\begin{array}{l}\text { Light PA: } \\
132 \\
\mathrm{~min} / \text { day, } \\
\text { MVPA: } \\
10.8 \\
\mathrm{~min} / \text { day }\end{array}$ & $\begin{array}{l}\text { Age, skin color, } \\
\text { schooling, economic } \\
\text { level, smoking, self- } \\
\text { perceived health, } \\
\text { number of morbidities, } \\
\text { and functional } \\
\text { capability. }\end{array}$ \\
\hline Health ABC study & $\begin{array}{l}\text { Manini, } \\
2006^{25}\end{array}$ & USA & 302 & 74.8 & 49.7 & 6.2 & 296 & $\begin{array}{l}\text { Doubly } \\
\text { labelled water }\end{array}$ & $\begin{array}{l}1.7 \\
\mathrm{kcal} / \text { day }\end{array}$ & $\begin{array}{l}\text { Age, sex, race study site, } \\
\text { weight, height, }\end{array}$ \\
\hline
\end{tabular}




\begin{tabular}{|c|c|c|c|c|c|c|c|c|c|c|}
\hline $\begin{array}{l}\text { Multicenter } \\
\text { Norwegian study }\end{array}$ & $\begin{array}{l}\text { Hansen, } \\
2020^{26}\end{array}$ & Norway & 2,183 & 57 & 47.0 & 9.1 & 60 & Accelerometer & $\begin{array}{l}\text { Median: } \\
8,002 \\
\text { steps/day }\end{array}$ & $\begin{array}{l}\text { Sex, wear time, VPA, } \\
\text { education, body mass } \\
\text { index, smoking, alcohol } \\
\text { intake, and number of } \\
\text { medical conditions. }\end{array}$ \\
\hline \multirow[t]{2}{*}{$\begin{array}{l}\text { NHANES (2003- } \\
2006)^{\mathrm{a}}\end{array}$} & $\begin{array}{l}\text { Borgundvaag, } \\
2016^{27}\end{array}$ & USA & 5,562 & 48.4 & 49.2 & 6.7 & 158 & Accelerometer & $\begin{array}{l}\text { bight PA: } \\
200.4 \\
\text { min/day, } \\
\text { MVPA: } \\
15.2 \\
\text { min/day }\end{array}$ & $\begin{array}{l}\text { Age, sex, race/ethnicity, } \\
\text { poverty-to-income ratio, } \\
\text { education, smoking, } \\
\text { alcohol, dietary fat, } \\
\text { dietary saturated fat, } \\
\text { dietary sodium, } \\
\text { accelerometer wear time, } \\
\text { and other physical } \\
\text { activity intensity }\end{array}$ \\
\hline & $\begin{array}{l}\text { Saint- } \\
\text { Maurice, } \\
2018^{28}\end{array}$ & USA & 4,840 & 57.0 & 49.7 & 6.6 & 219 & Accelerometer & $\begin{array}{l}\text { Light PA: } \\
4.1 \\
\text { hour/day, } \\
\text { MVPA: } \\
\text { 1.3hour/day }\end{array}$ & $\begin{array}{l}\text { Age, sex, race/ethnicity, } \\
\text { body mass index, } \\
\text { education, smoking, } \\
\text { alcohol consumption, } \\
\text { diabetes mellitus, stroke, } \\
\text { chronic heart failure, } \\
\text { reduced mobility, } \\
\text { cancer/ malignancy, and } \\
\text { either light PA or } \\
\text { MVPA }\end{array}$ \\
\hline
\end{tabular}




\begin{tabular}{|c|c|c|c|c|c|c|c|c|c|c|}
\hline $\begin{array}{l}\text { OPACH Study, an } \\
\text { ancillary study to the } \\
\text { Women's Health } \\
\text { Initiative }\end{array}$ & $\begin{array}{l}\text { Lamonte, } \\
2017^{10}\end{array}$ & USA & 6,382 & 78.6 & 0.0 & 3.1 & 218 & Accelerometer & $\begin{array}{l}\text { Total PA: } \\
334.1 \\
\text { min/day } \\
\text { (light PA: } \\
284.3 \\
\text { min/day, } \\
\text { MVPA: } \\
49.8 \\
\text { min/day) }\end{array}$ & $\begin{array}{l}\text { Age, race and ethnicity, } \\
\text { education, current } \\
\text { smoking, alcohol intake } \\
\text { in past } 3 \text { months, age at } \\
\text { menopause, self-rated } \\
\text { general health, number } \\
\text { of comorbid conditions, } \\
\text { accelerometer wear time } \\
\text { (h/d), and Short Physical } \\
\text { Performance Battery } \\
\text { score/MVPA/light PA }\end{array}$ \\
\hline $\begin{array}{l}\text { OPAL (Older People } \\
\text { and Active Living) }\end{array}$ & Fox, $2015^{19}$ & UK & 208 & $\geq 70$ & 51.2 & 4.2 & 378 & Accelerometer & $\begin{array}{l}64 \text { took } \\
<3,196 \\
\text { steps/day, } \\
67 \text { took } \\
3,196- \\
5,170 \\
\text { steps/day, } \\
\text { and } 70 \text { took } \\
>5,170 \\
\text { steps/day }\end{array}$ & $\begin{array}{l}\text { Age, sex, educational } \\
\text { attainment, Index of } \\
\text { Multiple Derivation, } \\
\text { weight status, GP } \\
\text { Management System, } \\
\text { number of self-reported } \\
\text { chronic illnesses at } \\
\text { baseline, and lower limb } \\
\text { function. }\end{array}$ \\
\hline $\begin{array}{l}\text { Osteoporotic } \\
\text { Fractures in Men } \\
\text { Study (MrOS) }\end{array}$ & $\begin{array}{l}\text { Ensrud, } \\
2014^{30}\end{array}$ & USA & 2,918 & 79.0 & 100.0 & 4.5 & 311 & $\begin{array}{l}\text { SenseWear } \\
\text { Pro Armband }\end{array}$ & $\begin{array}{l}\text { Light PA: } \\
67.7 \\
\text { min/day, at } \\
\text { least } \\
\text { moderate } \\
\text { PA: } 82.3 \\
\text { min/day }\end{array}$ & $\begin{array}{l}\text { Age, race, site, season, } \\
\text { education, marital status, } \\
\text { health status, smoking, } \\
\text { comorbidity burden, } \\
\text { depressive symptoms, } \\
\text { cognitive function, } \\
\text { number of instrumental } \\
\text { activity of daily living } \\
\text { impairments, percentage } \\
\text { body fat, PA scale for } \\
\text { the elderly, gait speed, } \\
\text { and time spent asleep }\end{array}$ \\
\hline $\begin{array}{l}\text { Study of Everyday } \\
\text { Physical Activity, a }\end{array}$ & $\begin{array}{l}\text { Chipperfield, } \\
2008^{11}\end{array}$ & Canada & 191 & 85.0 & 36.9 & 2.0 & 393 & Accelerometer & $\begin{array}{l}17.7 \% \text { were } \\
\text { moderately }\end{array}$ & $\begin{array}{l}\text { Age, functional status } \\
\text { (health-related }\end{array}$ \\
\hline
\end{tabular}


satellite study of the

Aging in Manitoba

Longitudinal Project

Sweden Attitude

Behaviour and

Change study

Tasped

Dwyer,

$2015^{12}$

Australia 2,576

58.8

47.6

$90 \%$

for 10

years

The British Regional Jefferis,

Heart Study $2018^{20}$

UK Biobank

Chudasama

$2019^{32}$

active and

$10.1 \%$ were

extremely

active

Accelerometer Light PA:

5 h 44

min/day,

MVPA: 31

$\min /$ day

850

8,856

steps/day restriction), physical

status (severity of

chronic conditions), and

psychological status

(positive affect)

Education, smoking

status, and presence of

hypertension, heart

disease, cancer, and

diabetes at baseline and

stratified by sex and age tertiles.

Age, sex, body mass

index at baseline, total

energy intake from all

sources $(\mathrm{kJ})$ at baseline,

current smoking status at

baseline, alcohol

consumption (g/day) at

baseline, education at

baseline and study

cohort

Age, region of residence,

season of wear,

min/day, accelerometer wear time,

MVPA: 40 social class, alcohol use,

min/day, smoking, sleep time,

mean living alone, body mass

steps/day: index, and mobility

4938

Accelerometer Median:

27.1

average

disability.

Age, sex, ethnicity,

socioeconomic status,

employment status,

acceleration education level, body

in $\mathrm{mg}$ /day mass index, smoking 


\begin{tabular}{|c|c|c|c|c|c|c|c|c|c|c|}
\hline $\begin{array}{l}\text { Women's Health } \\
\text { Study }\end{array}$ & Lee, $2019^{22}$ & USA & 16,741 & 72.0 & 0.0 & 4.3 & 70 & Accelerometer & $\begin{array}{l}5,499 \\
\text { steps/day }\end{array}$ & $\begin{array}{l}\text { and sedentary time. } \\
\text { Age, wear time, smoking } \\
\text { status, alcohol use, } \\
\text { intakes of saturated fat, } \\
\text { fiber, fruits, and } \\
\text { vegetables, hormone } \\
\text { therapy, parental history } \\
\text { of myocardial infarction, } \\
\text { family history of cancer, } \\
\text { general health, history of } \\
\text { cardiovascular disease, } \\
\text { history of cancer, cancer } \\
\text { screening, BMI, history } \\
\text { of hypertension, high } \\
\text { cholesterol, and diabetes. }\end{array}$ \\
\hline
\end{tabular}

${ }^{a}$ Out of 18 studies based on NHANES data only two studies were included in the meta-analysis (Borgundvaag, $2016^{27}$ for light physical activity and moderate-tovigorous physical activity; Saint-Maurice, $2018^{28}$ for total physical activity). Out of the two papers ${ }^{31,47}$ based on the Swedish Attitude Behaviour and Change study, Dohrn, $2017^{31}$ was included.

${ }^{b}$ Median

Abbreviations: PY=person-years, PA= physical activity, MVPA=moderate-to-vigorous physical activity

Out of 33 articles included in the review, only 15 were of high quality ${ }^{10,12,19,22,25,26,28,29,31,32,38,42,45-47}$ and the rest were moderate (Table 2). ${ }^{11,14 \text {, }}$

20,21,27,30,33-37,39-41,43,44,48,49, Out of 33 articles only 16 adjusted for comorbidities/self-rated health status or accounted for these in the analysis, ${ }^{10,11,19,22,25,26,28-32,45-49}$ the confounder that we deemed most important for quality assessment. 
Table 2. Quality of Studies Included in the Systematic Review (Newcastle Ottawa Scale)

\begin{tabular}{|c|c|c|c|c|c|c|c|c|c|}
\hline \multirow[t]{2}{*}{ Study } & \multicolumn{4}{|c|}{ Selection } & \multicolumn{2}{|l|}{ Comparability } & \multicolumn{2}{|l|}{ Outcome } & \multirow{2}{*}{$\begin{array}{l}\text { Total } \\
\text { stars } \\
\end{array}$} \\
\hline & $\begin{array}{l}\text { Represen } \\
\text { tativenes } \\
\text { S of the } \\
\text { exposed } \\
\text { cohort }\end{array}$ & $\begin{array}{l}\text { Selection } \\
\text { of non- } \\
\text { exposed } \\
\text { cohort }\end{array}$ & $\begin{array}{l}\text { Ascertainmen } \\
t \text { of exposure }\end{array}$ & $\begin{array}{l}\text { Outcome } \\
\text { not present } \\
\text { at start of } \\
\text { study }\end{array}$ & $\begin{array}{l}\text { Adjusted for } \\
\text { comorbidities/self- } \\
\text { rated health status } \\
\text { and other important } \\
\text { covariates }\end{array}$ & $\begin{array}{l}\text { Assessm } \\
\text { ent of } \\
\text { outcome }\end{array}$ & $\begin{array}{l}\text { Follow-up } \\
\text { long enough } \\
\text { for outcomes } \\
\text { to occur }\end{array}$ & $\begin{array}{l}\text { Adequa } \\
\text { cy of } \\
\text { follow } \\
\text { up }\end{array}$ & \\
\hline Beddhu, 2015 & $*$ & $*$ & $*$ & $*$ & $*$ & $*$ & & $*$ & 7 \\
\hline Bielemann, $2020^{49}$ & $*$ & $*$ & $*$ & $*$ & $* *$ & $*$ & & & 7 \\
\hline Borgundvaag, $2016^{27}$ & & $*$ & $*$ & $*$ & $*$ & $*$ & $*$ & $*$ & 7 \\
\hline Chipperfield, $2008^{11}$ & & $*$ & $*$ & $*$ & $* *$ & $*$ & & $*$ & 7 \\
\hline Chudasama, 2019 & $*$ & $*$ & $*$ & $*$ & $* *$ & $*$ & $*$ & $*$ & 9 \\
\hline Dohrn, $2017^{31}$ & & $*$ & $*$ & $*$ & $* *$ & $*$ & $*$ & $*$ & 8 \\
\hline Dwyer, $2015^{12}$ & $*$ & $*$ & $*$ & $*$ & $*$ & $*$ & $*$ & $*$ & 8 \\
\hline Edwards, $2016^{33}$ & & $*$ & $*$ & $*$ & $*$ & $*$ & $*$ & $*$ & 7 \\
\hline Ensrud, $2014^{30}$ & & $*$ & $*$ & $*$ & $* *$ & $*$ & & $*$ & 7 \\
\hline Evenson, $2017^{34}$ & & $*$ & $*$ & $*$ & $*$ & $*$ & $*$ & $*$ & 7 \\
\hline Evenson, $2016^{35}$ & & $*$ & $*$ & $*$ & $*$ & $*$ & $*$ & $*$ & 7 \\
\hline Fishman, $2016^{36}$ & & $*$ & $*$ & $*$ & $*$ & $*$ & $*$ & $*$ & 7 \\
\hline Fox, $2015^{19}$ & $*$ & $*$ & $*$ & $*$ & $* *$ & $*$ & & $*$ & 8 \\
\hline Hansen, $2020^{26}$ & $*$ & $*$ & $*$ & $*$ & $* *$ & $*$ & $*$ & $*$ & 9 \\
\hline Jefferis, $2018^{20}$ & & $*$ & $*$ & $*$ & $*$ & $*$ & $*$ & $*$ & 7 \\
\hline Koster, 2012 37 & & $*$ & $*$ & $*$ & $*$ & $*$ & & $*$ & 6 \\
\hline Lamonte, $2017^{10}$ & $*$ & $*$ & $*$ & $*$ & $* *$ & $*$ & & $*$ & 8 \\
\hline Lee, $2016^{38}$ & $*$ & $*$ & $*$ & $*$ & $*$ & $*$ & $*$ & $*$ & 8 \\
\hline Lee, $2019^{22}$ & $*$ & $*$ & $*$ & $*$ & $* *$ & $*$ & & $*$ & 8 \\
\hline Loprinzi \& Crush, $2016^{40}$ & & $*$ & $*$ & $*$ & $*$ & $*$ & $*$ & $*$ & 7 \\
\hline Loprinzi, $2017^{39}$ & & $*$ & $*$ & $*$ & $*$ & $*$ & $*$ & $*$ & 7 \\
\hline Loprinzi, $2016^{41}$ & & $*$ & $*$ & $*$ & $*$ & $*$ & $*$ & $*$ & 7 \\
\hline Manini, $2006^{25}$ & & $*$ & $*$ & $*$ & $* *$ & $*$ & $*$ & $*$ & 8 \\
\hline Matthews, $2016^{42}$ & $*$ & $*$ & $*$ & $*$ & $*$ & $*$ & $*$ & $*$ & 8 \\
\hline Saint-Maurice, $2018^{28}$ & $*$ & $*$ & $*$ & $*$ & $* *$ & $*$ & $*$ & $*$ & 9 \\
\hline Saint-Maurice, $2020^{29}$ & $*$ & $*$ & $*$ & $*$ & $* *$ & $*$ & $*$ & $*$ & 9 \\
\hline Schmid, $2015^{43}$ & & $*$ & $*$ & $*$ & $*$ & $*$ & & $*$ & 6 \\
\hline
\end{tabular}


Schmid, $2016^{44}$

Shiroma, $2019^{45}$

Tarp, $2020^{46}$

von Rosen, $2020^{47}$

Wanigatunga, $2019^{48}$

Yamamoto, $2018^{21}$

Notes: Representativeness of the exposed cohort: One star=Truly/somewhat representative of the general population

Selection of the non-exposed cohort: One star = Drawn from the same community as the exposed cohort

Comparability: One star $=$ Study controls for comorbidities/self-rated health status, $2^{\text {nd }}$ star $=$ Study controls for other confounders

Follow-up long enough for outcomes to occur: One star $=\geq 5$ years

Adequacy of follow up: Complete follow up/all groups had similar loss to follow-up/less than 5\% of total lost to follow-up 
Three $11,25,48$ out of the 15 cohort studies were not included in the meta-analyses because the dose of exposure could not be harmonised because they were defined differently. Manini et $\mathrm{al}^{25}$ reported a $57 \%$ lower risk for all cause mortality for individuals in highest compared with the lowest tertile (95\%CI, 0.21 to 0.88$)$. Chipperfield ${ }^{11}$ found moderately active individuals to have $49 \%$ lower odds of mortality compared to moderately inactive individuals. Likewise, extremely active individuals were found to have $70 \%$ lower odds of mortality compared to extremely inactive individuals. The Baltimore Longitudinal Study of Aging found every hour/day increase in total physical activity associated with $13 \%$ decreased risk for all cause mortality. ${ }^{48}$

\begin{tabular}{|c|c|c|c|}
\hline Author & $\begin{array}{l}\text { Number of } \\
\text { Deaths/Sample size }\end{array}$ & $\begin{array}{l}\text { Hazard } \\
\text { Ratio }(95 \% \mathrm{Cl})\end{array}$ & $\begin{array}{l}\% \\
\text { Weight }\end{array}$ \\
\hline \multicolumn{4}{|c|}{ Light Physical Activity (min/day) } \\
\hline Bielemann, 2020 (men) & $51 / 367$ & $0.26(0.07,0.95)$ & 4.36 \\
\hline Bielemann, 2020 (women) & $54 / 604$ & $0.09(0.01,0.67)$ & 1.80 \\
\hline Borgundvaag, 2016 & $578 / 5,562$ & $0.90(0.62,1.29)$ & 24.00 \\
\hline Ensrud, 2014 & $409 / 2,918$ & $0.64(0.44,0.93)$ & 23.61 \\
\hline Jefferis, 2018 & $194 / 1,274$ & $0.51(0.31,0.82)$ & 18.54 \\
\hline Lamonte, 2017 & $450 / 7,048$ & $0.57(0.42,0.76)$ & 27.69 \\
\hline Subtotal $(\mathrm{I}-$ squared $=49.3 \%$ & $=0.079)$ & $0.60(0.45,0.80)$ & 100.00 \\
\hline \multicolumn{4}{|c|}{ Moderate-to-Vigorous Physical Activity (min/day) } \\
\hline Bielemann, 2020 (men) & $51 / 367$ & $0.22(0.05,1.05)$ & 3.36 \\
\hline Bielemann, 2020 (women) & $54 / 604$ & $0.07(0.01,0.59)$ & 1.95 \\
\hline Borgundvaag, 2016 & $578 / 5,562$ & $0.22(0.11,0.44)$ & 12.00 \\
\hline Dohrn, 2017 & $79 / 851$ & $0.50(0.28,0.90)$ & 14.92 \\
\hline Ensrud, 2014 & $409 / 2,918$ & $0.63(0.44,0.91)$ & 23.46 \\
\hline Fox et al, 2015 & $33 / 213$ & $0.54(0.09,3.13)$ & 2.53 \\
\hline Jefferis, 2018 & $194 / 1,274$ & $0.52(0.31,0.87)$ & 17.15 \\
\hline Lamonte, 2017 & $450 / 7,048$ & $0.46(0.33,0.65)$ & 24.62 \\
\hline Subtotal $(\mathrm{I}$-squared $=39.8 \%$ & $=0.11)$ & $0.44(0.33,0.59)$ & 100.00 \\
\hline \multicolumn{4}{|l|}{ Total Physical Activity } \\
\hline Bielemann, 2020 (men)a $^{a}$ & $51 / 367$ & $0.23(0.06,0.84)$ & 3.35 \\
\hline Bielemann, 2020 (women)a & $54 / 604$ & $0.08(0.01,0.65)$ & 1.49 \\
\hline Chudasama, 2019b & $306 / 95,615$ & $0.29(0.19,0.46)$ & 12.83 \\
\hline Dohrn, 2017c & $79 / 851$ & $0.43(0.23,0.80)$ & 9.48 \\
\hline Dwyer, $2015^{d}$ & $219 / 2,576$ & $0.19(0.12,0.30)$ & 12.50 \\
\hline Fox, $2015^{d}$ & $33 / 213$ & $0.18(0.03,1.10)$ & 1.95 \\
\hline Hansen, $2020^{d}$ & $119 / 2,183$ & $0.42(0.21,0.84)$ & 8.43 \\
\hline Lamonte, 2017a & $450 / 7,048$ & $0.56(0.41,0.76)$ & 15.72 \\
\hline Lee et al., 2019d & $504 / 16,741$ & $0.34(0.24,0.48)$ & 14.88 \\
\hline Saint-Maurice, 2018a & $700 / 4,840$ & $0.26(0.16,0.44)$ & 11.55 \\
\hline Yamamoto, 2018d & $76 / 419$ & $0.46(0.22,0.96)$ & 7.84 \\
\hline Subtotal $(\mathrm{I}$-squared $=54.2 \%$ & $=0.016)$ & $0.33(0.25,0.43)$ & 100.00 \\
\hline \multicolumn{4}{|c|}{ NOTE: Weights are from random effects analysis } \\
\hline
\end{tabular}

Figure 1. Hazard ratio of all cause mortality for highest versus lowest category of light physical activity, moderate-to-vigorous physical activity, and total physical activity. Arrow indicates that the confidence interval extends beyond the width shown.

${ }^{\mathrm{a} U n i t}=\mathrm{min} /$ day; ${ }^{\mathrm{b}}$ milli-gravitational units (mg); ${ }^{\mathrm{c} U n i t=c o u n t s / d a y ;}{ }^{\mathrm{d}}$ Unit=steps/day 
There was moderate degree of heterogeneity in the studies included in the metaanalysis. Relatively high levels of light, moderate-to-vigorous, and total PA were each associated with reduced risk for all cause mortality (Figure 1). Total PA was found to be protective of all cause mortality with a pooled HR of 0.33 (95\% CI, 0.25 to 0.43 ).

Furthermore, compared to individuals who engaged in the lowest category of light PA, individuals in the highest category had $40 \%$ lower risk for all cause mortality. After including the outlier study for minutes/day of light PA, ${ }^{31}$ the mortality risk was reduced from an HR of 0.60 to 0.55 (Supplementary Figure 2). For MVPA, we observed a non-linear dose response association with all cause mortality $\left(\mathrm{P}_{\text {nonlinearity }}<0.001\right)$; the summary HR was lower at 0.44 (Figure 1) with no appreciable additional benefits beyond 40 minutes per day (Supplementary Figure 3).

The adjusted pooled HR of the sensitivity analysis for total PA by excluding five studies that measured steps/day ${ }^{12,19,21,22,26}$ instead of minutes/day was 0.34 (95\%CI, 0.23 to 0.51) (Supplementary Figure 4). When we analysed steps/day as a continuous measure, the pooled estimate showed that an extra 1,000 steps/day would reduce the risk of mortality by 13\% (Supplementary Figure 5). Minimal change to the results for studies that reported the estimates after exclusion of early deaths illustrates the robustness of the pooled HR for MVPA and total PA (Supplementary Figure 6).

Subgroup analyses showed that light PA was associated with $45 \%$ and $36 \%$ lower risk for mortality among women and men, respectively ( $p$-value= 0.634). For MVPA, these estimates were 63\% and 51\%, respectively (p-value $=0.626)($ Supplementary Figures 7 and 8). For light and moderate-to-vigorous PA, there was only one study that included participants with a mean age $\leq 60$ years. Therefore, we were unable to conduct analyses for this age group (Supplementary Figure 9). However, for total PA we found 74\% reduced risk for all cause mortality in participants with a mean age $\leq 60$ years; the corresponding risk was $60 \%$ in participants with a mean age greater than 60 years of age $(p$-value $=0.21)$ (Supplementary Figure 10). For light PA, a lone study was of high quality and the rest were of moderate quality. Subgroup analysis by study quality did not differ substantially from the overall estimate for MVPA (p-value =0.92) (Supplementary Figure 11) and for total PA (pvalue $=0.95)$ (Supplementary Figure 12). Estimates for $<5$ years versus $\geq 5$ years of follow-up did not differ much from the overall estimates especially for MVPA and total PA (Supplementary Figures 13, 14, and 15). We were able to assess publication bias for only MVPA (number of studies: 10). We found insufficient evidence for publication bias and small study effects (Egger's test p-value: 0.20) (Supplementary Figure 16). 


\section{DISCUSSION}

In this systematic review and meta-analysis of published cohort studies irrespective of the device (accelerometer, pedometer) and unit (minutes or hours/day, counts/minute, steps/day, or milli-gravitational units (mg)) used to measure PA, we found high versus low: light, moderate-to-vigorous, and total PA, were each associated with lower risk for all cause mortality. The findings from this systematic review of studies that used an objective measurement of PA are considerably lower than the pooled estimates obtained from the questionnaire-based studies. ${ }^{1,2}$

In addition to the six published studies that contributed data to the IPD meta-analysis by Ekelund et $\mathrm{al}^{9}$ we were able to synthesise evidence from nine additional studies. The results from the meta-analysis are consistent with that of Ekelund et $\mathrm{al}^{9}$ especially for total and moderate-to-vigorous PA, but that study included only accelerometer studies whereas for total PA we also included studies that used a pedometer to measure PA. A difference found in our meta-analysis was that the summary hazard ratio for MVPA was lower than for light PA when the highest category was compared to lowest category that warrants further investigation. Our results for light PA and MVPA are consistent with the results from a metaanalysis by Rojer et al. ${ }^{7}$

Woodcock et al ${ }^{1}$ in their systematic review based on questionnaires, reported $19 \%$ reduction in risk of mortality for 30 minutes daily of moderate intensity activity undertaken five days a week and a $24 \%$ reduction in risk for one hour/day. Samitz et al $^{2}$ estimated risk reductions of 14\% (30 min/day) and 26\% (one hour/day) for MVPA. In contrast, we found no evident additional benefits beyond 40 minutes of MVPA per day with a relatively stable hazard ratio around 0.50 .

For the highest compared to the lowest level of total PA, Samitz et $\mathrm{al}^{2}$ estimated an HR of 0.65 (95\%CI, 0.60 to 0.71 ) for all cause mortality. In our analysis of studies using objective measurement of PA, we found a pooled HR of 0.33 (95\%CI, 0.25 to 0.43 ) when comparing highest to lowest levels of total PA, that is a halving of the HR found in the questionnaire-based systematic review which reported the lowest HR for very high levels of PA. This finding is close to that reported by Ekelund et $\mathrm{al}^{9}$ wherein the hazard ratio was 0.27 when the highest quarter was compared to lowest quarter of accelerometer measured total PA expressed in counts/minute. The finding of a greater risk reduction in association with total PA versus MVPA when accelerometry is used is consistent with the view that total energy 
expenditure is the key determinant of reduced risk of mortality. Alternatively, the participants who undertake the highest quantity of total PA might be those who also are in the highest category for MVPA and who also take additional amounts of light activity.

Though only a modest number of studies were included in this review and metaanalysis, the sample size of most of the studies was reasonably large and the number of events in the meta- analysis was large $(n=3,903)$. A limitation of this study was the variability in the duration of PA across cohorts within the categories being compared, i.e. light, or moderate-to-vigorous PA. This, however, should not bias the key comparison made here between objective and subjective measurement of PA as such variability in duration of exposure within the different categories of PA must have been present in the questionnairebased studies even if the instrument did not capture it fully. Another limitation is the use of different objective measures of PA across studies. The meta-analysis for total PA included studies that used either accelerometer or pedometer. This should not be a major limitation as there is a high correlation $(\mathrm{r}=0.80)$ between accelerometers and pedometers in estimating total PA. ${ }^{50}$ Pedometers are unable to measure different intensities of $\mathrm{PA}^{50}$ and therefore only accelerometry data was available for studies that estimated light and moderate-to-vigorous PA. Even so, the definitions of PA intensity levels varies according to the cut-points used and the populations studied. ${ }^{51}$ Another limitation is that we included studies that measured steps/day either using pedometer or accelerometer that could be a source of measurement bias. However, two separate studies conducted among free living individuals (age range: 1860 years) found strong correlation between steps/day measured via accelerometer and pedometer..$^{-52}$

The comparison between results from cohorts using questionnaire and accelerometer could be criticised based on the grounds that they come from a different set of cohorts, involving potentially a different distribution of confounders, and variation in the prevalence of PA. However, given the similarity of the findings across both the studies using objective measurement and those using questionnaires, it is unlikely that the difference in estimates compared to the questionnaire-based studies could be explained by a form of sampling bias such as this. It is more likely that the difference in the overall magnitude of association observed between the objectively measured and questionnaire measured PA is real and can be accounted for by the use of a more accurate form of measurement. ${ }^{53}$ While we could not compare questionnaire-based estimates of risk to those estimated using objective measures in most of the studies included here, one of the studies based on NHANES data that was included in this systematic review ${ }^{35}$ reported an HR of 0.44 for accelerometer-assessed 
MVPA and all cause mortality when comparing the highest to lowest quartile. The corresponding estimate for self-reported MVPA was 0.63. Even though we did not present the data in our meta-analysis, the finding from a cohort study using doubly labelled water of a greater than $50 \%$ reduction in mortality risk further supports the conclusion that when objective measures are used a lower HR is likely to be seen. ${ }^{25}$

Another criticism might be that most of the studies in the current review used 7-day accelerometer data, while the questionnaire-based studies attempted to capture habitual activity and this might affect estimated associations. Arguing against this being an important cause of the differences observed is data from the Dwyer et al study ${ }^{12}$ which was able to examine associations using both baseline and repeat assessment with pedometer 3.7 years later. They found only a $4 \%$ difference in magnitude of the risk estimate for all cause mortality if the later measure was used rather than baseline, suggesting that one point of time measurement of PA reflects habitual activity reasonably well when used in cohort studies of this kind.

The protective effect in the studies using objective measurement appears to extend further into the upper range of PA than is seen with questionnaires. This could be due to greater measurement error at higher levels of PA with questionnaires. Supporting this possibility, a multi-centre study in Norway found participants reported more vigorous activity and less sedentary time in questionnaires than when PA was measured with an accelerometer. 54

Estimates of lower risk of death associated with PA in reviews based on both questionnaires and objective measures could be due to reverse causation. That is, individuals who were at a higher risk of death because of the presence of detectable or undetectable serious illness were less likely to engage in PA. This concern has been addressed in both study settings by the demonstration that removal of early deaths did not substantially affect the estimates of risk reduction especially for MVPA. However, it will require further investigation through more in-depth examination of health status at the time of assessment of PA in future cohorts. Mendelian randomisation, using PA as the exposure of interest might also become more feasible in the future and provide further insight into causality. ${ }^{55}$

\section{CONCLUSION}

The beneficial effect of PA estimated from cohort studies using objective measurement of PA is considerably higher than that obtained from the questionnaire-based studies. Although the focus of current guidelines is on moderate/vigorous PA it is apparent in this systematic 
review that the beneficial effect of PA extends across the spectrum of intensity levels. Newer cohort studies that have PA data measured by both accelerometer (with standardised cutpoints for intensity of PA) and questionnaire can potentially confirm the findings from this study. Examining not only mortality but also other outcomes such as cardiovascular diseases, type 2 diabetes, cancer, metabolic health, and mental health will be an important next step. 


\section{AUTHOR CONTRIBUTORSHIP}

RR acquired, analysed, and interpreted the data, drafted and revised the work critically for important intellectual content, and approved the final version. TD conceived the study, interpreted the data, drafted and revised the work critically for important intellectual content, and approved the final version. JH checked data abstractions and quality of the study, revised the work critically for important intellectual content, and approved the final version. AP, MW, KR, and SB interpreted the data, revised the work critically for important intellectual content, and approved the final version.

\section{DECLARATION OF INTERESTS}

All authors with the exception of KR declare no support from any organisation for the submitted work; no financial relationships with any organisations that might have an interest in the submitted work in the previous three years; no other relationships or activities that could appear to have influenced the submitted work. KR reports receipt of grants from the NIHR Biomedical Research Centre, Oxford Martin School, and RCUK (ESRC) during the conduct of the study, but declares no financial relationships with any organisations that might have an interest in the submitted work in the previous three years and no other relationships or activities that could appear to have influenced the submitted work.

\section{FUNDING SOURCES: None}




\section{REFERENCES}

1. Woodcock J, Franco OH, Orsini N, Roberts I. Non-vigorous physical activity and all-cause mortality: systematic review and meta-analysis of cohort studies. Int J Epidemiol. 2011;40(1):121-138. doi:10.1093/ije/dyq104

2. Samitz G, Egger M, Zwahlen M. Domains of physical activity and all-cause mortality: systematic review and dose-response meta-analysis of cohort studies. Int J Epidemiol. 2011;40(5):1382-1400. doi:10.1093/ije/dyr112

3. U.S. Department of Health and Human Service. 2008 Physical Activity Guidelines for Americans. https://health.gov/paguidelines/pdf/paguide.pdf. Published 2008.

4. Department of Health and Social Care, Llywodraeth Cymru Wlesh Government, Department of Health Northern Ireland and the Scottish Government. UK Chief Medical Officers' Physical Activity Guidelines . 2019.

doi:https://assets.publishing.service.gov.uk/government/uploads/system/uploads/attachment_d ata/file/832868/uk-chief-medical-officers-physical-activity-guidelines.pdf

5. Prince SA, Adamo KB, Hamel ME, Hardt J, Gorber SC, Tremblay M. A comparison of direct versus self-report measures for assessing physical activity in adults: a systematic review. Int $J$ Behav Nutr Phys Act. 2008;5:56. doi:10.1186/1479-5868-5-56

6. Lagerros YT, Lagiou P. Assessment of physical activity and energy expenditure in epidemiological research of chronic diseases. Eur J Epidemiol. 2007;22(6):353-362. doi:10.1007/s10654-007-9154-x

7. Rojer AGM, Ramsey KA, Trappenburg MC, et al. Instrumented measures of sedentary behaviour and physical activity are associated with mortality in community-dwelling older adults: A systematic review, meta-analysis and meta-regression analysis. Ageing Res Rev. 2020;61. doi:10.1016/j.arr.2020.101061

8. Hall KS, Hyde ET, Bassett DR, et al. Systematic review of the prospective association of daily step counts with risk of mortality, cardiovascular disease, and dysglycemia. Int J Behav Nutr Phys Act. 2020;17(1). doi:10.1186/s12966-020-00978-9 
9. Ekelund U, Tarp J, Steene-Johannessen J, et al. Dose-response associations between accelerometry measured physical activity and sedentary time and all cause mortality: systematic review and harmonised meta-analysis. BMJ. 2019;366:14570. doi:10.1136/bmj.14570

10. Lamonte MJ, Buchner DM, Rillamas-Sun E, et al. Accelerometer-measured physical activity and mortality in women aged 63 to 99. J Am Geriatr Soc. 2017. doi:10.1111/jgs.15201

11. Chipperfield JG. Everyday physical activity as a predictor of late-life mortality. Gerontologist. 2008;48(3):349-357. doi:10.1093/geront/48.3.349

12. Dwyer T, Pezic A, Sun C, et al. Objectively measured daily steps and subsequent long term all-cause mortality: The Tasped Prospective Cohort Study. PLoS One. 2015;10(11):e0141274e0141274. doi:10.1371/journal.pone.0141274

13. Tierney JF, Stewart LA, Ghersi D, Burdett S, Sydes MR. Practical methods for incorporating summary time-to-event data into meta-analysis. Trials. 2007;8:16. doi:10.1186/1745-6215-816

14. Beddhu S, Wei G, Marcus RL, Chonchol M, Greene T. Light-Intensity physical activities and mortality in the United States general population and CKD subpopulation. Clin J Am Soc Nephrol. 2015;10(7):1145 LP - 1153. doi:10.2215/CJN.08410814

15. Altman DG, Bland JM. How to obtain the confidence interval from a P value. BMJ Br Med J. 2011;343. http://www.bmj.com/content/343/bmj.d2090.abstract.

16. Hartling L, Milne A, Hamm MP, et al. Testing the Newcastle Ottawa Scale showed low reliability between individual reviewers. J Clin Epidemiol. 2013;66(9):982-993. doi:10.1016/j.jclinepi.2013.03.003

17. Wells GA, Shea B, O'Connell D, et al. The Newcastle-Ottawa Scale (NOS) for assessing the quality of nonrandomized studies in meta-analyses. http://www.ohri.ca/programs/clinical_epidemiology/oxford.asp. Published 2018.

18. DerSimonian R, Laird N. Meta-analysis in clinical trials. Control Clin Trials. 1986;7(3):177188. doi:10.1016/0197-2456(86)90046-2

19. Fox KR, Ku P-W, Hillsdon M, et al. Objectively assessed physical activity and lower limb 
function and prospective associations with mortality and newly diagnosed disease in UK older adults: an OPAL four-year follow-up study. Age Ageing. 2015;44(2):261-268.

doi:10.1093/ageing/afu168

20. Jefferis BJ, Parsons TJ, Sartini C, et al. Objectively measured physical activity, sedentary behaviour and all-cause mortality in older men: does volume of activity matter more than pattern of accumulation? Br J Sport Med. 2018. doi:10.1136/bjsports-2017-098733

21. Yamamoto N, Miyazaki H, Shimada M, et al. Daily step count and all-cause mortality in a sample of Japanese elderly people: A cohort study. BMC Public Health. 2018;18(1). doi:10.1186/s12889-018-5434-5

22. Lee IM, Shiroma EJ, Kamada M, Bassett DR, Matthews CE, Buring JE. Association of step volume and intensity with all-cause mortality in older women. JAMA Intern Med. 2019. doi:10.1001/jamainternmed.2019.0899

23. Orsini N, Li R, Wolk A, Khudyakov P, Spiegelman D. Meta-analysis for linear and nonlinear dose-response relations: examples, an evaluation of approximations, and software. Am J Epidemiol. 2012;175(1):66-73. doi:10.1093/aje/kwr265

24. Higgins JPT, Thompson SG, Deeks JJ, Altman DG. Measuring inconsistency in metaanalyses. BMJ Br Med J. 2003;327(7414):557-560. http://www.ncbi.nlm.nih.gov/pmc/articles/PMC192859/.

25. Manini TM, Everhart JE, Patel K V, et al. Daily activity energy expenditure and mortality among older adults. J Am Med Assoc. 2006;296(2):171-179. doi:10.1001/jama.296.2.171

26. Hansen BH, Dalene KE, Ekelund U, et al. Step by step: Association of device-measured daily steps with all-cause mortality—A prospective cohort Study. Scand J Med Sci Sports. 2020;30(9):1705-1711. doi:10.1111/sms.13726

27. Borgundvaag E, Janssen I. Objectively measured physical activity and mortality risk among American adults. Am J Prev Med. 2017;52(1):e25-e31. doi:10.1016/j.amepre.2016.09.017

28. Saint-Maurice PF, Troiano RP, Berrigan D, Kraus WE, Matthews CE. Volume of light versus moderate-to-vigorous physical activity: Similar benefits for all-cause mortality? J Am Heart Assoc. 2018;7(7):e008815. doi:http://dx.doi.org/10.1161/JAHA.117.008815 
29. Saint-Maurice PF, Troiano RP, Bassett DR, et al. Association of daily step count and step intensity with mortality among US adults. JAMA - J Am Med Assoc. 2020;323(12):1151-1160. doi:http://dx.doi.org/10.1001/jama.2020.1382

30. Ensrud KE, Blackwell TL, Cauley JA, et al. Objective measures of activity level and mortality in older men. J Am Geriatr Soc. 2014;62(11):2079-2087. doi:10.1111/jgs.13101

31. Dohrn I-M, Sjöström M, Kwak L, Oja P, Hagströmer M. Accelerometer-measured sedentary time and physical activity-A 15 year follow-up of mortality in a Swedish population-based cohort. J Sci Med Sport. 2018;21(7):702-707. doi:10.1016/j.jsams.2017.10.035

32. Chudasama Y V, Khunti KK, Zaccardi F, et al. Physical activity, multimorbidity, and life expectancy: a UK Biobank longitudinal study. BMC Med. 2019;17(1):108. doi:10.1186/s12916-019-1339-0

33. Edwards MK, Loprinzi PD. All-cause mortality risk as a function of sedentary behavior, moderate-to-vigorous physical activity and cardiorespiratory fitness. Phys Sportsmed. 2016;44(3):223-230. doi:10.1080/00913847.2016.1221751

34. Evenson KR, Herring AH, Wen F. Accelerometry-assessed latent class patterns of physical activity and sedentary behavior with mortality. Am J Prev Med. 2017;52(2):135-143. doi:https://doi.org/10.1016/j.amepre.2016.10.033

35. Evenson KR, Wen F, Herring AH. Associations of accelerometry-assessed and self-reported physical activity and sedentary behavior with all-cause and cardiovascular mortality among US adults. Am J Epidemiol. 2016;184(9):621-632. doi:10.1093/aje/kww070

36. Fishman EI, Steeves JA, Zipunnikov V, et al. Association between objectively measured physical activity and mortality in NHANES. Med Sci Sport Exerc. 2016;48(7):1303-1311. doi:http://dx.doi.org/10.1249/MSS.0000000000000885

37. Koster A, Caserotti P, Patel K V, et al. Association of sedentary time with mortality independent of moderate to vigorous physical activity. PLoS One. 2012;7(6):e37696. doi:10.1371/journal.pone.0037696

38. Lee PH. Examining non-linear associations between accelerometer-measured physical activity, sedentary behavior, and all-cause mortality using segmented Cox regression. Front Physiol. 
2016;7:272. doi:10.3389/fphys.2016.00272

39. Loprinzi PD. Light-Intensity Physical activity and all-cause mortality. Am J Heal Promot. 2017;31(4):340-342. doi:10.4278/ajhp.150515-ARB-882

40. Loprinzi PD, Crush E. Sensory impairment, functional balance and physical activity with allcause mortality. J Phys Act Heal. 2016;13(9):980-987.

doi:http://dx.doi.org/10.1123/jpah.2015-0692

41. Loprinzi PD, Loenneke JP, Ahmed HM, Blaha MJ. Joint effects of objectively-measured sedentary time and physical activity on all-cause mortality. Prev Med. 2016;90:47-51. doi:http://dx.doi.org/10.1016/j.ypmed.2016.06.026

42. Matthews CE, Keadle SK, Troiano RP, et al. Accelerometer-measured dose-response for physical activity, sedentary time, and mortality in US adults. Am J Clin Nutr. 2016;104(5):1424-1432. doi:10.3945/ajcn.116.135129

43. Schmid D, Ricci C, Leitzmann MF. Associations of objectively assessed physical activity and sedentary time with all-cause mortality in US adults: the NHANES study. PLoS One. 2015;10(3):e0119591. doi:http://dx.doi.org/10.1371/journal.pone.0119591

44. Schmid D, Ricci C, Baumeister SE, Leitzmann MF. Replacing sedentary time with physical activity in relation to mortality. Med Sci Sport Exerc. 2016;48(7):1312-1319. doi:http://dx.doi.org/10.1249/MSS.0000000000000913

45. Shiroma EJ, Lee IM, Schepps MA, Kamada M, Harris TB. Physical ACTIVITY PATTERNS AND MORtality: The weekend warrior and activity bouts. Med Sci Sport Exerc. 2019;51(1):35-40. http://search.ebscohost.com/login.aspx?direct=true \&db=sph\&AN=133566867\&site=ehostlive\&authtype=ip,uid.

46. Tarp J, Hansen BH, Fagerland MW, Steene-Johannessen J, Anderssen SA, Ekelund U. Accelerometer-measured physical activity and sedentary time in a cohort of US adults followed for up to 13 years: The influence of removing early follow-up on associations with mortality. Int J Behav Nutr Phys Act. 2020;17(1):39. doi:http://dx.doi.org/10.1186/s12966020-00945-4 
47. von Rosen P, Dohrn IM, Hagstromer M. Latent profiles analysis of physical activity and sedentary behaviour with mortality risk: a 15-year follow-up. Scand J Med Sci Sport. 2020. doi:http://dx.doi.org/10.1111/sms.13761

48. Wanigatunga AA, Di J, Zipunnikov V, et al. Association of total daily physical activity and fragmented physical activity with mortality in older adults. JAMA Network Open. 2019;2(10):e1912352. doi:10.1001/jamanetworkopen.2019.12352

49. Bielemann RM, LaCroix AZ, Bertoldi AD, et al. Objectively measured physical activity reduces the risk of mortality among Brazilian older adults. J Am Geriatr Soc. 2020;68(1):137146. doi:10.1111/jgs. 16180

50. Tudor-Locke C, Ainsworth BE, Thompson RW, Matthews CE. Comparison of pedometer and accelerometer measures of free-living physical activity. Med Sci Sport Exerc. 2002;34(12):2045-2051. doi:10.1249/01.Mss.0000039300.76400.16

51. Barnett A, van den Hoek D, Barnett D, Cerin E. Measuring moderate-intensity walking in older adults using the ActiGraph accelerometer. BMC Geriatr. 2016;16(1):211. doi:10.1186/s12877-016-0380-5

52. Behrens TK, Dinger MK. Comparisons of accelerometer and pedometer determined steps in free living samples. J Phys Act Health. 2011;8(3):390-397. doi:10.1123/jpah.8.3.390

53. Tudor-Locke C, Johnson WD, Katzmarzyk PT. Accelerometer-determined steps per day in US adults. Med Sci Sports Exerc. 2009;41(7):1384-1391. doi:10.1249/MSS.0b013e318199885c

54. Dyrstad SM, Hansen BH, Holme IM, Anderssen SA. Comparison of self-reported versus accelerometer-measured physical activity. Med Sci Sport Exerc. 2014;46(1):99-106. doi:10.1249/MSS.0b013e3182a0595f

55. Smith GD, Ebrahim S. Mendelian Randomization: Genetic Variants as Instruments for Strengthening causal inference in observational studies. National Research Council (US) Committee on Advances in Collecting and Utilizing Biological Indicators and Genetic Information in Social Science Surveys; Weinstein M Wachter KW VJW, ed. Biosoc Surv. 2008;(16). https://www.ncbi.nlm.nih.gov/books/NBK62433/. 